
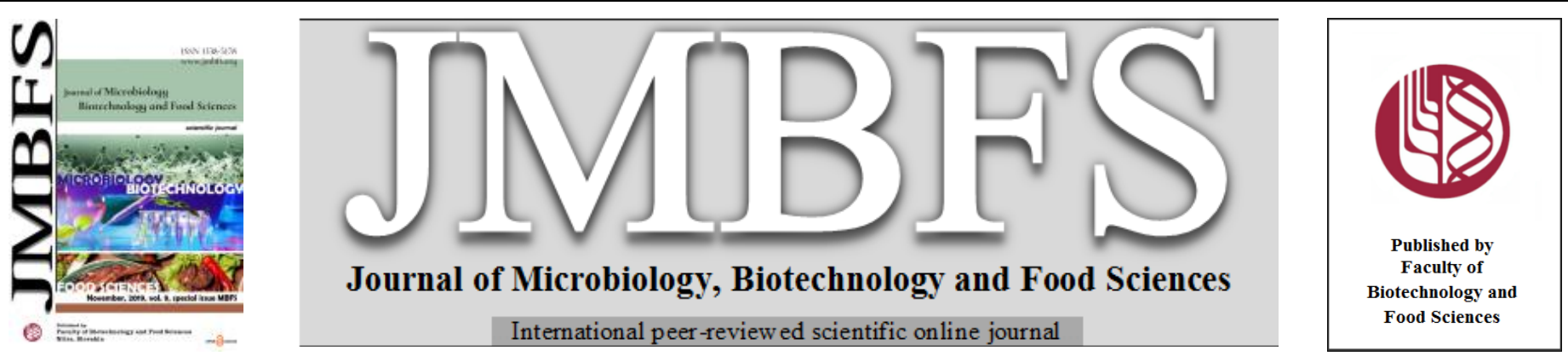

\title{
DIVERSITY OF BACTERIA IN SLOVAK AND FOREIGN HONEY, WITH ASSESSMENT OF ITS PHYSICO- CHEMICAL QUALITY AND COUNTS OF CULTIVABLE MICROORGANISMS
}

\author{
Vladimíra Kňazovickál, Michal Gábor², Martina Miluchová2, Marek Bobko³, Juraj Medo*4
}

Address(es): Ing. Juraj Medo, PhD.

${ }^{1}$ National Agricultural and Food Centre, Research Institute for Animal Production Nitra, Institute of Apiculture Liptovsky Hradok, Gasperikova 599,03380 Liptovsky Hradok, Slovakia.

${ }^{2}$ Slovak University of Agriculture in Nitra, Faculty of Agrobiology and Food Resources, Department of Genetics and Animal Breeding Biology, Tr. A. Hlinku 2, 94976 Nitra, Slovakia.

${ }^{3}$ Slovak University of Agriculture in Nitra, Faculty of Biotechnology and Food Sciences, Department of Technology and Quality of Animal Products, Tr. A. Hlinku 2, 94976 Nitra, Slovakia.

${ }^{4}$ Slovak University of Agriculture in Nitra, Faculty of Biotechnology and Food Sciences, Department of Microbiology, Tr. A. Hlinku 2, 94976 Nitra, Slovakia, phone number: +421376415810.

*Corresponding author: juraj.medo@uniag.sk

doi: 10.15414/jmbfs.2019.9.special.414-421

\section{ARTICLE INFO}

Received 23. 8. 2019

Revised 7. 10. 2019

Accepted 10. 10. 2019

Published 8. 11. 2019

Regular article open $\partial_{\text {aCcess }}$

\begin{abstract}
The aim of the study was to assess microbial microbiome of 30 honey samples and compare potential differences between the samples from apiaries and commercial trade in Slovakia and foreign countries (Latvia, Switzerland, India, Japan and Tanzania) as well as to indicate their physico-chemical and basic microbiological quality. Study of each sample consisted of physico-chemical analysis (water content, $\mathrm{pH}$, free acidity and electrical conductivity), basic microbiological analysis performed by dilution plating method (total plate count, sporulating aerobic microorganisms, bacteria from Enterobacteriaceae family, preliminary lactic acid bacteria and microscopic fungi) and metagenomic analysis for bacterial diversity evaluation. Seven samples did not meet with legislative limits of physico-chemical parameters. Average values of cultivable microbial groups ranged at level 1-2 log CFU. $\mathrm{g}^{-1}$, while bacteria from Enterobacteriaceae family were not detected in any samples. Sporulating microorganisms occurred most often, in $77 \%$ of samples and yeasts were proven in $60 \%$ of samples. Bacterial diversity, determined by metagenomic analysis, was varied. We distinguished 2 groups - group A and group B. Group A contained mainly fresh (produced in 2018) Slovak and Swiss honey and we found mainly genus Lactobacillus followed by genus Bombella in them. Group B contained mainly older Slovak honey (produced in 2017) and commercial foreign samples, in which production year is difficult to know. Group B samples were interesting because of human bacteria presence with genus Prevotella dominance. Redundancy analysis showed significant connection of the electric conductivity and microbial assemblage, that indicates important influence of botanical origin to microbial representation in honey.
\end{abstract}

Keywords: Bee product, Electrical conductivity, Lactic acid bacteria, Metagenomics, Prevotella sp.

\section{INTRODUCTION}

Honey is a natural product of bees traditionally used as food product and medicine all over the world including Slovakia, Romania and Russia (Guziy et al., 2017; Šedík et al., 2019). Consumption of honey almost doubled in last decade in Slovakia. Comparing with other food, ripe high quality honey is considered to be a food with a minimal level of microbial contamination with many beneficia effects in human nutrition and with no or limited risks for human health. Most of the potential microorganisms relevant for food safety are expected to be in inactive forms as they cannot survive in honey because of its properties including hyperosmolarity (Bovo et al., 2018). Essentially, honey is a supersaturated solution comprising approximately $80 \%$ sugars by weight, predominantly fructose and glucose, with sucrose, maltose, and many other sugars at much lower concentrations (Cooper, 2014). Israili (2014) concluded antimicrobial activity of honey as follows: A large number of in vitro and limited clinical studies have confirmed the broad-spectrum antimicrobial (antibacterial, antifungal, antivira and antimycobacterial) properties of honey, which may be attributed to the acidity (presence of acids, low $\mathrm{pH}$ ), osmotic effect, high sugar concentration, presence of bacteriostatic and bactericidal factors (hydrogen peroxide, antioxidants, lysozyme, polyphenols, phenolic acids, flavonoids, methylglyoxal and bee peptides) and increase in cytokine release and to immune modulating and anti-inflammatory properties of honey.

In general, sporulating bacteria and yeast belong to common inhabitants of honey. Martins et al. (2003) tested 80 multifloral honey samples from retail public markets in Portugal; they were centred on Bacillaceae spores and fungi; spores of Clostridium perfringens were not detected in any sample, Bacillus cereus were identified in $13.7 \%$; yeasts and microscopic filamentous fungi (MFF) were detected in $88.8 \%$ samples with identification of 3 MFF genera: Aspergillus Penicillium and Mucor and 2 genera of yeasts: Saccharomyces and Candida. Tolba et al. (2007) identified bacilli in 7 honey samples mainly from Northern Ireland. They found Bacillus pumilus, B. licheniformis, B. subtilis, B. fusiformis and Paenibacillus motobuensis. Amir et al. (2010) analysed occurrence of MFF in 19 honeys of blossom, blended and honeydew origin from Algeria. Microscopic filamentous fungi were found mainly in samples with low water content (16.2 and $17 \%$ ) and included it that this fact was influenced by xerophilic properties of honey and MFF. Honey, especially in the fresh state, is interesting by presence of lactic acid bacteria (LAB) presence. For the first time Olofsson et Vásquez (2008) found LAB in the honey stomach (crop) and in the fresh honey as well, and they suggested that honey can be considered a fermented food product because of the LAB involved in honey production. In the last time, numerous studies about microorganisms in bees were published with main focus to bee intestinal microbiome. Maes et al. (2016) performed bee-cage experiments with different diet and demonstrated that typically occurring alterations in diet quality play a significant role in colony health and establishment of a dysbiotic gut microbiome. According to Bonilla-Rosso et Engel (2018), simple sugars such as glucose and fructose present in nectar and pollen, and complex polysaccharides such as pectin from the pollen wall are apparent substrates for bacterial fermentation. 
Properties as well as quantity and diversity of microorganisms of honey are influenced by many factors. Some of them can be partially or fully managed during the production process while others not. Snowdon et Cliver (1996) described primary sources of microorganisms in honey, including pollen, the digestive tract of honey bees, dust, air, dirt and flowers as well as secondary contamination, which can be taken place during and after honey extraction, including humans, equipment, containers, wind, dust, insects, animals or water. Blossom honey comes from nectar of plants and honeydew honey is originated from honeydew produced mainly by aphids. Gilliam et al. (1983) found that nectar is not a major source of microorganisms for honey bees, but nectars of some flowers, they tested, contained Staphylococcus sp. as well as gram-positive and gram-negative rod-shaped bacteria, actinomycetes and fungi. Bacteria are probably added during the process by which nectar becomes honey and the nectar sugars probably act as inducers for the resident microbiota in honey stomach (crop), with enhancing their numbers (Olofsson et Vásquez, 2008). Honeybees collect honeydew, which is product from aphids inhabiting green parts of plants and at the same time, with honeydew, they may collect other attached structures, such as the hyphae or fungal spores of plant pathogens and microalgae (Escuredo et al., 2012).

According to Snowdon et Cliver (1996), bacteria or yeasts are principally found in comb honey and sometimes also filamentous fungi are present, while information about presence and persistence of viruses and parasites are not available. However some human enteric viruses, such as hepatitis A, sustain dry conditions and could be expected to persist in honey.

Traditionally number of microorganisms particularly bacteria and microscopic fungi were assessed by cultivation of them on artificially prepared media in laboratory. However such conditions are not responded to requirement of many microbial species. Microbiology has experienced a transformation during the last decade that has altered microbiologists' view of microorganisms and how to study them (Handelsman, 2004). At present, methods based on DNA analysing are more available for scientists. Metagenomic analysis, particularly $16 \mathrm{~S}$ rRNA gene sequencing on high throughput sequencing platform Illumina became the most common and accurate analyses. These techniques were previously used to asses gut microbiomes of bees. Bovo et al. (2018) analysed 2 honey samples (orange tree blossom and eucalyptus honey from beekeepers in Sicily) by shotgun metagenomics and surprisingly they noted that the largest number of reads assigned to 5 organism group (Viruses, Bacteria, Plants, Fungi and Arthropods) matched virus sequences in both honey samples $(67.55-98.56 \%)$. Microbiology of honey is still full of secretes and it indicates, that honey is probably able to keep DNA or RNA of various organisms.
The aim of the study was to analyse the honey samples in term of their basic physico-chemical properties, counts of cultivable microorganisms and metagenomic analysis to detect diversity of bacteria.

\section{MATERIAL AND METHODS}

\section{Material}

Totally, we tested 30 honey samples: 24 were available in Slovakia (18 with Slovak origin and 1 with origin in EU and non-EU countries) and 6 were obtained from foreign countries: from Latvia (mix EU and non-EU countries honey), Switzerland India, Japan and Tanzania. From botanical point of view, 24 samples were blossom (4 false acacia, 1 rape, 1 sunflower, 1 buckwheat and 17 multifloral), 5 samples were honeydew and 1 sample was blended. According to way of obtaining, 24 samples were from apiaries (22 directly from beekeepers and 2 from farmers' market), and 6 samples were from commercial trade. Detailed characterization is in the table 1 .

\section{Physico-chemical analysis}

The physico-chemical analysis consisted of water content, $\mathrm{pH}$, free acidity and electrical conductivity. These parameters were measured according IHC (2009): water content by refractometric method, potentiometric $\mathrm{pH}$ measurement by $\mathrm{pH}$ meter, free acidity by titration to $\mathrm{pH} 8.3$ and electrical conductivity (EC) by conductometric method. Individual measurements were performed at least 2 times with average expression.

As the electrical conductivity is one of main descriptive criteria to differentiate blossom and honeydew honey where honeydew (and nectar only of some plants, e. g. chestnut) is linked to higher conductivity we sorted samples to the three groups as follows (table 1):

- Low EC - honeys with EC to 0.29 mS.cm ${ }^{-1}$ (n = 13, EC: $0.11-0.29$ $\left.\mathrm{mS} . \mathrm{cm}^{-1}\right)$,

- $\quad$ Middle EC - honeys with EC from 0.30 to $0.69 \mathbf{~ m S . c m}^{-1}(\mathrm{n}=10$, EC $0.32-0.69 \mathrm{mS} . \mathrm{cm}^{-1}$ )

- Higher EC - honeys with EC from $\mathbf{0 . 7 0} \mathbf{~ m S . c m}{ }^{-1}(\mathrm{n}=7$, EC: 0.72 $\left.1.32 \mathrm{mS} . \mathrm{cm}^{-1}\right)$.

Table 1 Characterization of analysed samples

\begin{tabular}{|c|c|c|c|c|c|}
\hline Sample code & Botanical origin & Geographical origin & Obtaining & $\begin{array}{c}\text { Year of } \\
\text { production }\end{array}$ & Note \\
\hline BloSCo1 & $\begin{array}{l}\text { blossom (false acacia - } \\
\text { Robinia pseudoacacia) }\end{array}$ & Slovakia & $\begin{array}{c}\text { commercial trade } \\
\text { (Slovakia) }\end{array}$ & 2018 & \\
\hline BloSBee1 & $\begin{array}{l}\text { blossom (rape - } \\
\text { Brassica napus) }\end{array}$ & $\begin{array}{l}\text { western Slovakia } \\
\text { (Male Krstenany) }\end{array}$ & directly from beekeeper & 2017 & creamed \\
\hline BloSBee2 & blossom & $\begin{array}{l}\text { middle Slovakia } \\
\text { (Dolny Pajer) }\end{array}$ & directly from beekeeper & 2018 & \\
\hline BloSBee3 & $\begin{array}{l}\text { blossom (with dominance of } \\
\text { false acacia) }\end{array}$ & $\begin{array}{l}\text { western Slovakia } \\
\text { (Dezerice) }\end{array}$ & directly from beekeeper & 2018 & $1^{\text {st }}$ extraction \\
\hline BloFCo1 & blossom & $\begin{array}{l}\text { mix EU and non-EU } \\
\text { honey }\end{array}$ & $\begin{array}{c}\text { commercial trade } \\
\text { (Slovakia) }\end{array}$ & \multicolumn{2}{|c|}{ best before 2020} \\
\hline BloSBee4 & blossom (false acacia) & $\begin{array}{c}\text { western Slovakia } \\
\text { (Nitra-castle, Cerman) }\end{array}$ & directly from beekeeper & 2018 & $1^{\text {st }}$ extraction \\
\hline BloFCo2 & blossom & $\begin{array}{l}\text { mix EU and non-EU } \\
\text { honey }\end{array}$ & $\begin{array}{l}\text { commercial trade } \\
\text { (Latvia) }\end{array}$ & \multicolumn{2}{|c|}{ best before 2019} \\
\hline BloSBee5 & $\begin{array}{l}\text { blossom (with dominance of } \\
\text { false acacia) }\end{array}$ & $\begin{array}{c}\text { eastern Slovakia } \\
\text { (Sobrance) }\end{array}$ & directly from beekeeper & \multicolumn{2}{|l|}{2017} \\
\hline BloSBee6 & blossom & $\begin{array}{l}\text { middle Slovakia } \\
\text { (Smrecany) }\end{array}$ & directly from beekeeper & 2018 & $1^{\text {st }}$ extraction \\
\hline BloSBee7 & blossom & $\begin{array}{l}\text { middle Slovakia } \\
\text { (Horna Orava) }\end{array}$ & directly from beekeeper & \multicolumn{2}{|l|}{2018} \\
\hline BloFCo3 & blossom & India (Himalaya) & commercial trade (India) & \multicolumn{2}{|c|}{ durability 2014 - 2017} \\
\hline BloSBee8 & blossom & middle Slovakia (Babin) & directly from beekeeper & \multicolumn{2}{|l|}{2018} \\
\hline BloSBee9 & blossom & $\begin{array}{l}\text { middle Slovakia } \\
\text { (Smrecany) }\end{array}$ & directly from beekeeper & 2018 & $2^{\text {nd }}$ extraction \\
\hline BloSBee10 & $\begin{array}{c}\text { blossom (sunflower - } \\
\text { Helianthus annus) }\end{array}$ & $\begin{array}{l}\text { western Slovakia } \\
\text { (Dunajska Luzna) }\end{array}$ & directly from beekeeper & \multicolumn{2}{|l|}{2018} \\
\hline BloFCo4 & blossom & $\begin{array}{c}\text { Japan (labelled as } \\
\text { Mexican Orange honey) }\end{array}$ & commercial trade (Japan) & \multicolumn{2}{|c|}{ best before 2024} \\
\hline BloHoSBee1 & blended & $\begin{array}{l}\text { middle Slovakia } \\
\text { (Smrecany) }\end{array}$ & directly from beekeeper & 2017 & $4^{\text {th }}$ extracting \\
\hline BloSBee11 & $\begin{array}{l}\text { blossom (buckwheat - } \\
\text { Fagopyrum esculentum) }\end{array}$ & $\begin{array}{l}\text { western Slovakia } \\
\text { (Risnovce) }\end{array}$ & directly from beekeeper & 2018 & \\
\hline
\end{tabular}




\begin{tabular}{|c|c|c|c|c|c|}
\hline BloFBee1 & blossom & Switzerland & $\begin{array}{c}\text { directly from beekeeper } \\
\text { (farmers' market) }\end{array}$ & 2018 & $\begin{array}{c}\text { slightly } \\
\text { fermented }\end{array}$ \\
\hline BloSBee12 & blossom (spring) & $\begin{array}{l}\text { eastern Slovakia } \\
\text { (Nova Lubovna) }\end{array}$ & $\begin{array}{c}\text { directly from beekeeper } \\
\text { (Switzerland) }\end{array}$ & 2018 & \\
\hline HoSBee1 & honeydew & eastern Slovakia (Kosice) & directly from beekeeper & 2017 & $\begin{array}{c}\text { suspected of } \\
\text { adulteration }\end{array}$ \\
\hline BloSBee13 & blossom & $\begin{array}{c}\text { western Slovakia } \\
\text { (Dezerice) }\end{array}$ & $\begin{array}{c}\text { directly from beekeeper } \\
\text { (farmers' market) }\end{array}$ & 2018 & $2^{\text {nd }}$ extraction \\
\hline BloSBee14 & $\begin{array}{c}\text { blossom (raspberry - Rubus } \\
\text { idaeus, linden - Tilia sp.) }\end{array}$ & eastern Slovakia (Orlov) & directly from beekeeper & 2018 & \\
\hline BloSBee15 & blossom & $\begin{array}{l}\text { western Slovakia } \\
\text { (Nitra-Zobor) }\end{array}$ & directly from beekeeper & 2018 & $3^{\text {rd }}$ extraction \\
\hline BloSBee16 & $\begin{array}{l}\text { blossom (dandelion - Taraxacum } \\
\text { officinale, willow - Salix sp.) }\end{array}$ & eastern Slovakia (Orlov) & directly from beekeeper & 2018 & \\
\hline BloFBee2 & blossom & Switzerland & directly from beekeeper & 2018 & $\begin{array}{l}\text { slightly } \\
\text { fermented }\end{array}$ \\
\hline HoSBee2 & honeydew & eastern Slovakia (Orlov) & $\begin{array}{c}\text { directly from beekeeper } \\
\text { (Switzerland) }\end{array}$ & 2018 & \\
\hline HoSBee3 & honeydew & middle Slovakia (Sutovo) & directly from beekeeper & 2017 & \\
\hline HoSBee4 & honeydew & $\begin{array}{l}\text { eastern Slovakia } \\
\text { (Nova Lubovna) }\end{array}$ & directly from beekeeper & 2018 & \\
\hline HoSBee5 & honeydew & western Slovakia (Nitra) & directly from beekeeper & 2018 & $5^{\text {th }}$ extraction \\
\hline BloFCo5 & $\begin{array}{c}\text { blossom } \\
\text { (October blossom) }\end{array}$ & Tanzania (Kasalu) & directly from beekeeper & dura & $2016-2019$ \\
\hline
\end{tabular}

sample code (according to botanical, geographical origin and obtaining): first part: Blo - blossom, Ho - honeydew, BloHo - blended (blossom-honeydew); second

part: S - Slovakian, F - foreign; third part: Bee - from beekeeper, Co - from commercial trade; EU - European union, western Slovakia - Bratislava Region, Nitra

Region, Trnava Region, Trencin Region; middle Slovakia - Banska Bystrica Region, Zilina Region, eastern Slovakia - Kosice Region, Presov Region

\section{Microbiological analysis - dilution plating method}

We used dilution plating method to quantify the microorganisms in honey. The target microbial groups were: TPC (total plate count), SAM (sporulating aerobic microorganisms), bacteria from Enterobacteriaceae family, preliminary LAB (lactic acid bacteria) and MF (microscopic fungi, i. e. yeasts and MFF microscopic filamentous fungi). The basic dilution $\left(10^{-1}\right)$ was performed by homogenizing $5 \mathrm{~g}$ honey and $45 \mathrm{ml}$ saline solution $(0.85 \% \mathrm{NaCl}, 0.10 \%$ peptone $)$ Specific conditions of microbiological analysis are listed in the table 2 .

Table 2 Quantitative microbial examination of honey

\begin{tabular}{|c|c|c|c|c|c|}
\hline \multirow{2}{*}{ Microbial group } & \multirow{2}{*}{ Medium } & \multirow{2}{*}{ Inoculation } & \multicolumn{3}{|l|}{ Cultivation } \\
\hline & & & temperature & time & $\mathrm{O}_{2}$ requirement \\
\hline TPC & GTY & pouring & $30^{\circ} \mathrm{C}$ & $2-3$ days & aerobic \\
\hline SAM & NA 2 & pouring* & $25^{\circ} \mathrm{C}$ & 3 days & aerobic \\
\hline Enterobacteriaceae & VRBG & pouring & $30^{\circ} \mathrm{C}$ & $1-2$ days & aerobic \\
\hline preliminary LAB & MRS & pouring** & $37^{\circ} \mathrm{C}$ & 3 days & aerobic** $^{*}$ \\
\hline MF & DG 18 & pouring & $25^{\circ} \mathrm{C}$ & 5-7 days & aerobic \\
\hline
\end{tabular}

TPC - total plate count, cultivated on GTY - agar with glucose, tryptone and yeast extract (HiMedia ${ }^{\circledR}$, India); SAM - sporulating aerobic microorganisms, noculated by *pouring - after heat shock (at $80^{\circ} \mathrm{C}$ for $10 \mathrm{~min}$ ), cultivated on NA 2 - nutrient agar no. 2 (HiMedia ${ }^{\circledR}$ India); Enterobacteriaceae family, cultivated on VRBG - violet red bile glucose agar (HiMedia ${ }^{\circledR}$. India); LAB - lactic acid bacteria, inoculated by **double-pouring (decrease of oxygen in medium), cultivated on MRS - de Man, Rogosa and Sharpe agar (HiMedia ${ }^{\circledR}$, India); MF - microscopic fungi, cultivated on DG 18 - Dichloran Glycerol agar - with chloramphenicol (HiMedia ${ }^{\circledR}$, India)

\section{DNA extraction}

We weighted $20 \mathrm{~g}$ of honey to sterile $50 \mathrm{ml}$ tube and added sterile distilled wate to total volume of $45 \mathrm{ml}$. The solution was heated at $75{ }^{\circ} \mathrm{C}$ and rotated in hybridization chamber to solve the honey. Then, samples were centrifuged at 8000 $\mathrm{rpm}$ for $10 \mathrm{~min}$. Supernatant was removed and pellet was resuspended in $1 \mathrm{x}$ PBS solution ( $\mathrm{pH}$ 7.4), which was added to total volume $30 \mathrm{ml}$. Then, samples were centrifuged at $8000 \mathrm{rpm}$ for $5 \mathrm{~min}$. Most of supernatant was removed. We left only approximately $2 \mathrm{ml}$ and transferred it to $2 \mathrm{ml}$ tubes. These tubes were again centrifuged at $13000 \mathrm{rpm}$ for $5 \mathrm{~min}$ and supernatant was removed. Pellets were stored at $-80{ }^{\circ} \mathrm{C}$ to next analysis. To pellet we added glass beads and $250 \mu$ PrepMan ${ }^{\mathrm{TM}}$ Ultra Sample Preparation Reagent (ThermoFisher Scientific) and then homogenized by bead homogenizer BeadBugTM 3 (Benchmark Scientific) at highest speed for $1 \mathrm{~min}$. We heated the samples at $110^{\circ} \mathrm{C}$ for $5 \mathrm{~min}$. After heating, the tubes were centrifuged and lysates were transferred to new tube and used for PCR reaction

\section{Metagenomic analysis}

Barcoded primers $515 \mathrm{~F}$ and $806 \mathrm{R}$ (Caporaso et al., 2011) which amplify V4 section of the $16 \mathrm{~S}$ gene were used for PCR reaction. The composition of the PCR mixture was as follows: $15 \mu$ l KAPA HIFI HotStart mix 2X (Kapa Biosystems), 8 $\mu 1$ of each primer with a concentration of $2.5 \mu \mathrm{M}$, and $1 \mu \mathrm{l}$ of isolated DNA. Amplification was performed using SureCycler 8800 Thermal Cycler (Agilent) and thermal profile was following: Initial denaturation for $90 \mathrm{~s}$ at $98{ }^{\circ} \mathrm{C}$ followed by 35 cycles of denaturation for $15 \mathrm{~s}$ at $98{ }^{\circ} \mathrm{C}$, annealing for $15 \mathrm{~s}$ at $62{ }^{\circ} \mathrm{C}$ and extension for $15 \mathrm{~s}$ at $72{ }^{\circ} \mathrm{C}$. Final extension was $2 \mathrm{~min}$ at $72{ }^{\circ} \mathrm{C}$. PCR products were purified using a PCR purification kit (Jena Bioscience), quantified by qubit (Invitrogen), diluted to the same concentration and pooled together. Illumina sequencing library was prepared by TruSeq LT PCR free kit (Illumina) with a modification involving omission of the DNA fragmentation and size selection. NebNext Quantification kit (New England Biolabs) was used for the library quantification then the library was diluted to $4 \mathrm{nM}$ concentration, and denatured. The sequencing reaction was performed on Illumina MiSeq using the MiSec Reagent Kit v3 (600-cycle).

Acquired sequencing data was processed in SEED environment (Větrovský $\boldsymbol{e t}$ al., 2018). Forward and reverse reads were joined with minimum 100 base overlap. Only sequences with quality higher than Q30 were used in further analysis. Sequences were assigned to samples according used barcodes and then barcoded primers were removed. Sequences were checked for chimeras and clustered to operational taxonomic unit (OTUs) using Vsearch (Rognes et al., 2016) at a similarity level of $97 \%$. From each cluster (OTU) the most abundant sequence was found and identified using RDP classifier (Wang et al., 2007). Chloroplast sequences originated from pollen were removed.

\section{Statistical analysis}

Data from microbiological analysis were calculated as $\log$ CFU.g ${ }^{-1}$. Significan difference was assessed if it was at least $1.00 \log \mathrm{CFU} \cdot \mathrm{g}^{-1}$. Data from cultivation analysis as well as metagenomic data were descriptively processed in MS Excel 2007.

Shannon and Chaol diversity indices were calculated using ComEcolPaC (Drozd,

2010). For analysis of microbial communities and their connection to physicchemical parameters RDA was derived and significance were analysed by 
permutation test in package Vegan (Oksanen et al., 2013) in $\mathrm{R}$ statistical environment (Team R, 2013). OTUs with an only single member were removed prior these analyses.

Heatmaps were made using Heatmap3 (Zhao et al., 2014) in $R$

\section{RESULTS AND DISCUSSION}

\section{Physico-chemical and microbiological quality}

In the table 3, there are results of basic physico-chemical parameters and microbial counts for each sample. Water content ranged from 15.0 to $20.7 \%$ with average value $17.5 \pm 1.3 \%$. Similar results were published by Bušová et Kouřimská (2018), who found average water content $18.3 \%$ in honey directly from Czech beekeepers and $18.6 \%$ in commercial samples. Water is a parameter, which is important for evaluation of honey maturity and its low value relates directly with long durability. According to Council Directive 2001/110/EC, honey has to contain less than $20 \%$ of water. Only one sample (BloFBee1) exceeded this limit. The sample was gently fermented before analysis. According to Bogdanov et Martin (2002) the water content of honey can naturally be as low as $13.6 \%$ and as high as $23.0 \%$ depending on source of the honey, climatic conditions and other factors. Water in honey is related to honey preservation and storage, as high water content can lead to a growth of yeasts and microscopic filamentous fungi, causing fermentation, flavour loss and low shelf life (Al-Farsi et al., 2018). Fermentation does not usually a problem in honey with water content less than 18\% (Bogdanov et Martin, 2002).

Table 3 Physico-chemical parameters, microbial counts and diversity of microbial assemblage of tested honey samples

\begin{tabular}{|c|c|c|c|c|c|c|c|c|c|c|c|}
\hline \multirow{2}{*}{$\begin{array}{l}\text { Sample } \\
\text { code }\end{array}$} & \multicolumn{4}{|c|}{ Physico-chemical quality } & \multicolumn{6}{|c|}{ Microbiological quality $\left(\log \mathrm{CFU} \cdot \mathrm{g}^{-1}\right)$} & \multirow{2}{*}{$\begin{array}{c}\text { Diversity } \\
\begin{array}{c}\text { Shannon's } \\
\text { index }\end{array}\end{array}$} \\
\hline & $\begin{array}{c}\text { Water } \\
(\%)\end{array}$ & pH & $\begin{array}{c}\text { FA } \\
\left(\text { meq.kg }{ }^{-1}\right) \\
\end{array}$ & $\begin{array}{c}\mathbf{E C} \\
\left(\mathrm{mS} . \mathrm{cm}^{-1}\right) \\
\end{array}$ & TPC & SAM & $\mathbf{E}$ & pLAB & yeasts & MFF & \\
\hline BloSCo1 & 16.3 & 4.1 & 13.2 & 0.11 & 3.15 & 2.57 & $<1.00$ & $<1.00$ & $<1.00$ & $<1.00$ & 0.459 \\
\hline BloSBee1 & 16.1 & 3.8 & 19.1 & 0.13 & 1.85 & $<1.00$ & $<1.00$ & $<1.00$ & $<1.00$ & $<1.00$ & 6.659 \\
\hline BloSBee2 & 17.7 & 3.8 & 20.2 & 0.16 & $<1.00$ & $<1.00$ & $<1.00$ & $<1.00$ & $<1.00$ & $<1.00$ & 5.514 \\
\hline BloSBee3 & 18.5 & 3.8 & 18.2 & 0.17 & 2.28 & 2.30 & $<1.00$ & $<1.00$ & 1.00 & $<1.00$ & 5.198 \\
\hline BloFCo1 & 17.4 & 4.7 & 7.65 & 0.18 & 1.60 & $<1.00$ & $<1.00$ & $<1.00$ & $<1.00$ & $<1.00$ & 6.325 \\
\hline BloSBee4 & 17.6 & 3.8 & 18.8 & 0.20 & $<1.00$ & 1.60 & $<1.00$ & $<1.00$ & 1.30 & 1.00 & 4.662 \\
\hline BloFCo2 & 17.8 & 4.4 & 17.2 & 0.20 & 2.00 & 1.30 & $<1.00$ & $<1.00$ & & & 7.098 \\
\hline BloSBee5 & 16.9 & 4.1 & 28.5 & 0.22 & $<1.00$ & $<1.00$ & $<1.00$ & $<1.00$ & $<1.00$ & $<1.00$ & 7.077 \\
\hline BloSBee6 & 18.6 & 3.9 & 18.0 & 0.23 & 4.17 & 1.70 & $<1.00$ & 3.57 & 2.00 & 2.04 & 4.007 \\
\hline BloSBee7 & 19.9 & 3.5 & 48.0 & 0.26 & 1.00 & 1.00 & $<1.00$ & $<1.00$ & 1.48 & 1.00 & 4.760 \\
\hline BloFCo3 & 16.4 & 4.2 & 10.0 & 0.26 & 2.19 & 2.10 & $<1.00$ & 1.60 & 1.00 & $<1.00$ & 6.632 \\
\hline BloSBee8 & 18.6 & 3.3 & 31.3 & 0.28 & 1.48 & $<1.00$ & $<1.00$ & $<1.00$ & 2.00 & 1.00 & 3.719 \\
\hline BloSBee9 & 18.0 & 3.8 & 20.6 & 0.29 & 2.37 & 1.48 & $<1.00$ & 3.08 & 2.07 & 1.30 & 5.760 \\
\hline $\begin{array}{r}\text { LOW EC } \\
\text { mean } \pm \text { SD }\end{array}$ & $\begin{array}{c}n=13 \\
\mathbf{1 7 . 7} \pm \mathbf{1 . 1}\end{array}$ & $\begin{array}{c}\mathrm{n}=13 \\
\mathbf{3 . 9} \pm \mathbf{0 . 4}\end{array}$ & $\begin{array}{c}\mathrm{n}=13 \\
\mathbf{2 0 . 8} \pm \mathbf{1 0 . 4}\end{array}$ & $\begin{array}{c}\mathrm{n}=13 \\
\mathbf{0 . 2 1} \pm \mathbf{0 . 0 6}\end{array}$ & $\begin{array}{c}\mathrm{n}=10 \\
\mathbf{2 . 2 1} \pm \mathbf{0 . 9 0}\end{array}$ & $\begin{array}{c}\mathrm{n}=8 \\
1.76 \pm 0.53\end{array}$ & ND & $\begin{aligned} \mathrm{n} & =3 \\
2.75 & \pm 1.03\end{aligned}$ & $\begin{aligned} n & =7 \\
1.55 & \pm 0.47\end{aligned}$ & $\begin{aligned} n & =5 \\
1.27 & \pm 0.45\end{aligned}$ & $\begin{array}{c}\mathrm{n}=13 \\
\mathbf{5 . 2 2} \pm \mathbf{1 . 8 1}\end{array}$ \\
\hline BloSBee10 & 15.0 & 3.7 & 29.4 & 0.32 & $<1.00$ & 1.48 & $<1.00$ & $<1.00$ & $<1.00$ & $<1.00$ & 4.261 \\
\hline BloFCo4 & 18.6 & 4.3 & 52.0 & 0.35 & 1.90 & 1.60 & $<1.00$ & $<1.00$ & & & 5.761 \\
\hline BloHoSBee1 & 18.3 & 3.8 & 54.5 & 0.36 & 3.58 & 1.00 & $<1.00$ & 3.54 & 1.30 & $<1.00$ & 6.956 \\
\hline BloSBee11 & 16.0 & 3.6 & 40.0 & 0.37 & 2.11 & $<1.00$ & $<1.00$ & $<1.00$ & $<1.00$ & $<1.00$ & 6.046 \\
\hline BloFBee1 & 20.7 & 4.0 & 25.2 & 0.42 & 1.48 & 1.48 & $<1.00$ & $<1.00$ & 1.00 & $<1.00$ & 2.127 \\
\hline BloSBee12 & 16.9 & 4.4 & 19.1 & 0.43 & 1.30 & 1.30 & $<1.00$ & $<1.00$ & 1.00 & $<1.00$ & 3.179 \\
\hline HoSBee1 & 16.2 & 3.8 & 42.7 & 0.49 & 1.00 & 1.00 & $<1.00$ & $<1.00$ & $<1.00$ & $<1.00$ & 6.989 \\
\hline BloSBee13 & 17.6 & 3.7 & 40.9 & 0.51 & 2.26 & 1.70 & $<1.00$ & $<1.00$ & $<1.00$ & $<1.00$ & 3.729 \\
\hline BloSBee14 & 18.2 & 3.7 & 42.3 & 0.64 & 1.95 & $<1.00$ & $<1.00$ & $<1.00$ & 1.70 & $<1.00$ & 1.774 \\
\hline BloSBee15 & 16.6 & 3.8 & 41.3 & 0.69 & 2.48 & 1.30 & $<1.00$ & $<1.00$ & 2.53 & 1.30 & 3.294 \\
\hline $\begin{array}{c}\text { MIDDLE EC } \\
\text { mean } \pm \text { SD }\end{array}$ & $\begin{array}{c}\mathrm{n}=10 \\
\mathbf{1 7 . 4} \pm \mathbf{1 . 6}\end{array}$ & $\begin{array}{c}\mathrm{n}=10 \\
\mathbf{3 . 9} \pm \mathbf{0 . 3}\end{array}$ & $\begin{array}{c}\mathrm{n}=10 \\
\mathbf{3 8 . 7} \pm \mathbf{1 1 . 2}\end{array}$ & $\begin{array}{c}n=10 \\
\mathbf{0 . 4 6} \pm \mathbf{0 . 1 3}\end{array}$ & $\begin{aligned} n & =9 \\
\mathbf{2 . 0 1} & \pm \mathbf{0 . 7 6}\end{aligned}$ & $\begin{aligned} \mathrm{n} & =8 \\
\mathbf{1 . 3 6} & \pm \mathbf{0 . 2 6}\end{aligned}$ & ND & $\begin{array}{l}\mathrm{n}=1 \\
3.54 \\
\end{array}$ & $\begin{array}{c}n=5 \\
1.51 \pm 0.64\end{array}$ & $\begin{array}{l}\mathrm{n}=1 \\
\mathbf{1 . 3 0} \\
\end{array}$ & $\begin{array}{c}\mathrm{n}=10 \\
\mathbf{4 . 4 1} \pm \mathbf{1 . 9 2}\end{array}$ \\
\hline BloSBee16 & 17.6 & 3.6 & 57.0 & 0.72 & 2.24 & 1.30 & $<1.00$ & $<1.00$ & 3.07 & 1.30 & 2.067 \\
\hline BloFBee2 & 18.2 & 3.8 & 74.3 & 0.80 & 1.60 & 2.21 & $<1.00$ & $<1.00$ & 1.00 & $<1.00$ & 3.355 \\
\hline HoSBee2 & 17.1 & 4.2 & 45.1 & 0.88 & 2.30 & 1.30 & $<1.00$ & $<1.00$ & 2.21 & $<1.00$ & 1.857 \\
\hline HoSBee3 & 15.2 & 4.7 & 16.0 & 1.00 & 1.30 & 1.60 & $<1.00$ & $<1.00$ & 1.00 & $<1.00$ & 3.357 \\
\hline HoSBee4 & 17.2 & 4.3 & 48.2 & 1.04 & 1.70 & 1.00 & $<1.00$ & $<1.00$ & 1.95 & $<1.00$ & 2.438 \\
\hline HoSBee5 & 16.4 & 4.5 & 68.0 & 1.20 & 2.10 & 1.70 & $<1.00$ & $<1.00$ & 2.00 & $<1.00$ & 4.838 \\
\hline BloFCo5 & 18.0 & 4.6 & 25.2 & 1.32 & 2.26 & 1.60 & $<1.00$ & 1.70 & & & 5.835 \\
\hline $\begin{array}{c}\text { HIGHER EC } \\
\text { mean } \pm \mathrm{SD}\end{array}$ & $\begin{array}{c}\mathrm{n}=7 \\
\mathbf{1 7 . 1} \pm \mathbf{1 . 0}\end{array}$ & $\begin{array}{c}\mathrm{n}=7 \\
\mathbf{4 . 2} \pm \mathbf{0 . 4}\end{array}$ & $\begin{array}{c}n=7 \\
47.7 \pm 21.3\end{array}$ & $\begin{array}{c}\mathrm{n}=7 \\
0.99 \pm \mathbf{0 . 2 1}\end{array}$ & $\begin{aligned} \mathrm{n} & =7 \\
1.93 & \pm 0.39\end{aligned}$ & $\begin{array}{c}\mathrm{n}=7 \\
\mathbf{1 . 5 3} \pm \mathbf{0 . 3 8}\end{array}$ & ND & $\begin{array}{l}\mathrm{n}=1 \\
\mathbf{1 . 7 0} \\
\end{array}$ & $\begin{array}{c}n=6 \\
1.87 \pm 0.79\end{array}$ & $\begin{array}{l}\mathrm{n}=1 \\
\mathbf{1 . 3 0} \\
\end{array}$ & $\begin{array}{c}\mathrm{n}=7 \\
\mathbf{3 . 3 9} \pm \mathbf{1 . 4 8}\end{array}$ \\
\hline
\end{tabular}

sample code (according to botanical, geographical origin and obtaining): first part: Blo - blossom, Ho - honeydew, BloHo - blended (blossom-honeydew); second part: S - Slovakian, F - foreign; third part: Bee - from beekeeper, Co - from commercial trade;

FA - free acidity, EC - electrical conductivity; TPC - total plate count, SAM - sporulating aerobic microorganisms, E - bacteria from Enterobacteriaceae family, pLAB - preliminary lactic acid bacteria, MFF - microscopic filamentous fungi, SD - standard deviation, $n$ - number of samples with detected value, ND - not detected

Values of $\mathrm{pH}$ ranged from 3.3 to 4.7 with average value $4.0 \pm 0.4$. Similarly, Amir et al. (2010) found $\mathrm{pH}$ of Algerian honey from 3.7 to 4.7, with average $\mathrm{pH} 4.0$. All honeys are acidic with a pH-value generally lying between 3.5 and 5.5 , due to the presence of organic acids that contribute to honey flavour and stability against microbial spoilage (Bogdanov et al., 2004). Organic acids constitute $0.5 \%$ of honey and include gluconic acid which is bye product of enzymatic break down of glucose (Olaitan et al., 2007). According to Escuredo et al. (2012) $\mathrm{pH}$ and electrical conductivity are two parameters widely used to distinguish between nectar and honeydew honeys, but they found $\mathrm{pH}$ from 3.5 to 5.0 without significant differences between blossom and honeydew honeys from Galicia (Nortwest
Spain). Al-Farsi et al. (2018) tested 58 Omani honey samples and found wider range of $\mathrm{pH}$ - from 3.46 to 7.51, while they stated that in Oman there are two main sources for honey - summer plant Acacia tortilis (from Fabaceae family) and winter plant Ziziphus spina-Christi, L., called Sidr (from Rhamnaceae family). Value of $\mathrm{pH}$ as well as total acidity and free acidity have some classification power for the discrimination between unifloral, while lactones, due to their strong variability, do not provide useful information (Bogdanov et al., 2004). Overall, free acidity of tested samples ranged from 7.7 to $74.3 \mathrm{meq} \cdot \mathrm{kg}^{-1}$. According to Council Directive 2001/110/EC, honey has to contain not more than 50 meq. $\mathrm{kg}^{-1}$. Five samples ( 2 from middle EC group and 3 from higher EC group) exceeded the 
limit. Average values of free acidity were different depending on electrical conductivity (EC) dividing: $20.8 \pm 10.4$ meq. $\mathrm{kg}^{-1}$ in honeys with low EC, $38.7 \pm$ 11.2 meq. $\mathrm{kg}^{-1}$ in honeys with middle $\mathrm{EC}$ and $47.7 \pm 21.3 \mathrm{meq} \cdot \mathrm{kg}^{-1}$. In general, blossom honey posses lower free acidity comparing with honeydew honey, except lime, buckwheat, eucalyptus honey or some other blossom honey. Zielińska et al. (2014) found free acidity $14.7 \pm 4.6$ meq. $\mathrm{kg}^{-1}$ in rape (Brassica napus), $15.4 \pm 6.6$ meq. $\mathrm{kg}^{-1}$ in multifloral, $30.7 \pm 17.4$ meq. $\mathrm{kg}^{-1}$ in lime (Tilia sp.), $29.8 \pm 11.8$ meq.kg ${ }^{1}$ in goldenrod, $35.6 \pm 8.5$ meq. $\mathrm{kg}^{-1}$ in honeydew and $45.0 \pm 6.4 \mathrm{meq} \cdot \mathrm{kg}^{-1}$ in buckwheat (Fagopyrum esculentum) Polish honey.

Electrical conductivity (EC) in tested honey ranged from 0.11 to $1.32 \mathrm{mS}^{-\mathrm{cm}^{-1}}$. Low EC was $0.21 \pm 0.06 \mathrm{mS} . \mathrm{cm}^{-1}$ on average and these honeys were mainly from false acacia, rape or blossom - multifloral honey. Middle EC was $0.46 \pm 0.13$ $\mathrm{mS} . \mathrm{cm}^{-1}$ on average and these honeys were from sunflower, buckwheat, raspberry, linden, blossom - multifloral or blended. Sample HoSBee1 was labelled as honeydew honey. We found EC $0.49 \mathrm{mS} . \mathrm{cm}^{-1}$, what is not sufficient for EC of honeydew honey. According to Council Directive 2001/110/EC, EC of honeydew honey has to be not less than $0.8 \mathrm{mS} . \mathrm{cm}^{-1}$. EC correlates well with the mineral content of honey (Bogdanov et al., 2004). Minerals are present in honey in very small quantities $(0.17 \%)$ with potassium as the most abundant (Olaitan et al., 2007). Minerals in honeydew as well as chestnut honey are higher comparing with blossom honey. Escuredo et al. (2012) determined average EC of blossom honey $0.540 \pm 0.200 \mathrm{mS} . \mathrm{cm}^{-1}$ (with range: $0.224-0.920 \mathrm{mS} . \mathrm{cm}^{-1}$ ) and total minerals of blossom honey $148.6 \pm 55.9$ mg. $\mathrm{kg}^{-1}$ (with range: $47.9-280.3 \mathrm{mg} \cdot \mathrm{kg}^{-1}$ ); and average EC of honeydew honey $0.830 \pm 0.200 \mathrm{mS}^{-\mathrm{cm}^{-1}}$ (with range $0.482-1.168$ $\mathrm{mS} . \mathrm{cm}^{-1}$ ) and total minerals $235.4 \pm 80.8 \mathrm{mg} . \mathrm{kg}^{-1}$ (with range $95.2-387.4 \mathrm{mg} . \mathrm{kg}$ $\left.{ }^{1}\right)$. Amir et al. (2010) found of Algerian honey - ash content 0.13-1.02\%, with average $0.4 \%$ and electrical conductivity $0.3-1.2 \mathrm{mS} . \mathrm{cm}^{-1}$, with average 0.6 $\mathrm{mS} . \mathrm{cm}^{-1}$ and indicated the honeys as blossom (63\%), honeydew (16\%) and blended $(21 \%)$. The electrical conductivity is good criterion related to botanical origin of honey and thus is very often used in routine honey control instead of the ash content (Gulfraz et al., 2011).

We detected viable microbial counts as follows: TPC (87\% of samples) $>$ SAM ( $77 \%$ of samples) $>$ yeasts $(60 \%$ of samples) $>$ MFF $(23 \%$ of samples $)>$ pLAB (17\% of samples) $>$ bacteria from Enterobacteriaceae family ( $0 \%$ of samples) Target microbial groups ranged from ND (not detected, i. e. less than $1.00 \mathrm{log}$ CFU.g ${ }^{-1}$ ) to $4.17 \log$ CFU.g ${ }^{-1}$ (table 3). Average values of tested microbial groups were at level $1-2 \log$ CFU.g ${ }^{-1}$. Tolba et al. (2007) demonstrated total viable counts from $<100$ to 1700 CFU.g ${ }^{-1}\left(<2.00\right.$ to $3.23 \mathrm{log}$ CFU.g $\left.{ }^{-1}\right)$ and did not find yeasts or MFF. Sporulating bacteria, MFF and yeasts are typically found in honey, often at low numbers, while spores can persist indefinitely (Snowdon $\boldsymbol{e t}$ Cliver, 1996).

\section{Diversity of bacteria}

Totally 155310 of high quality and chimera-free sequences were obtained representing average 5177 sequences per sample. Sequences were clustered into 11926 clusters and 1743 of them had at least 5 members and while 5630 OTUs were presented by single sequence. A part of sequences, 323 OTUs comprising $11517(7.5 \%)$ sequences were identified as chloroplast 16S rRNA genes Chloroplast co-amplification is common issue when plant tissue samples are analysed (Medo et al., 2018). In our samples chloroplasts sequences were linked to pollen grains naturally occurring in honey.

There were found occurrence of microbial species from 18 phyla (figure 1). The most common phylum was Firmicutes followed by Proteobacteria and Bacteroidetes and Actinobacteria. Other phyla were significantly less common. There was apparent difference between samples in occurrence of Bacteroidetes. Differences between samples were most apparent on genus level which is the lowest taxonomic level with fully reliable identification for the used method. Diversity of bacteria expressed as values of Shannon's index shoved values in very wide range 0.46 to 7.10 (Table 3 ). Dominance of single or few species in a sample resulted to very low diversity indices. Analysis of sequences identified 492 genera however only 52 had more than $2 \%$ share in any sample (Figure 2).

According to Bovo et al. (2018), bacteria from honey could be group combining their main role/action or prevalent ecological niche and their putative origin: Predominant group consists of bacteria typical for the hive micro-environment (also common microbiota of the bee gut), e. g. Lactobacillus kunkeei (obligate fructophilic LAB), Parasaccharibacter apium, Gilliamella apicola, Frischella perrara. Bee pathogens, e. g. Melissococcus plutonius or Paenibacillus larvae represent the second group. The third abundant group of microbes in honey are plant associated species, e. g. Pseudomonas syringae, Erwinia amylovora, Spiroplasma citri. Ubiquitous and specialized species, e. g. Escherichia coli, Bacillus cereus as well as antagonistic bacteria like Pseudomonas agglomerans (with action against E. amylovora) are also common in honey.

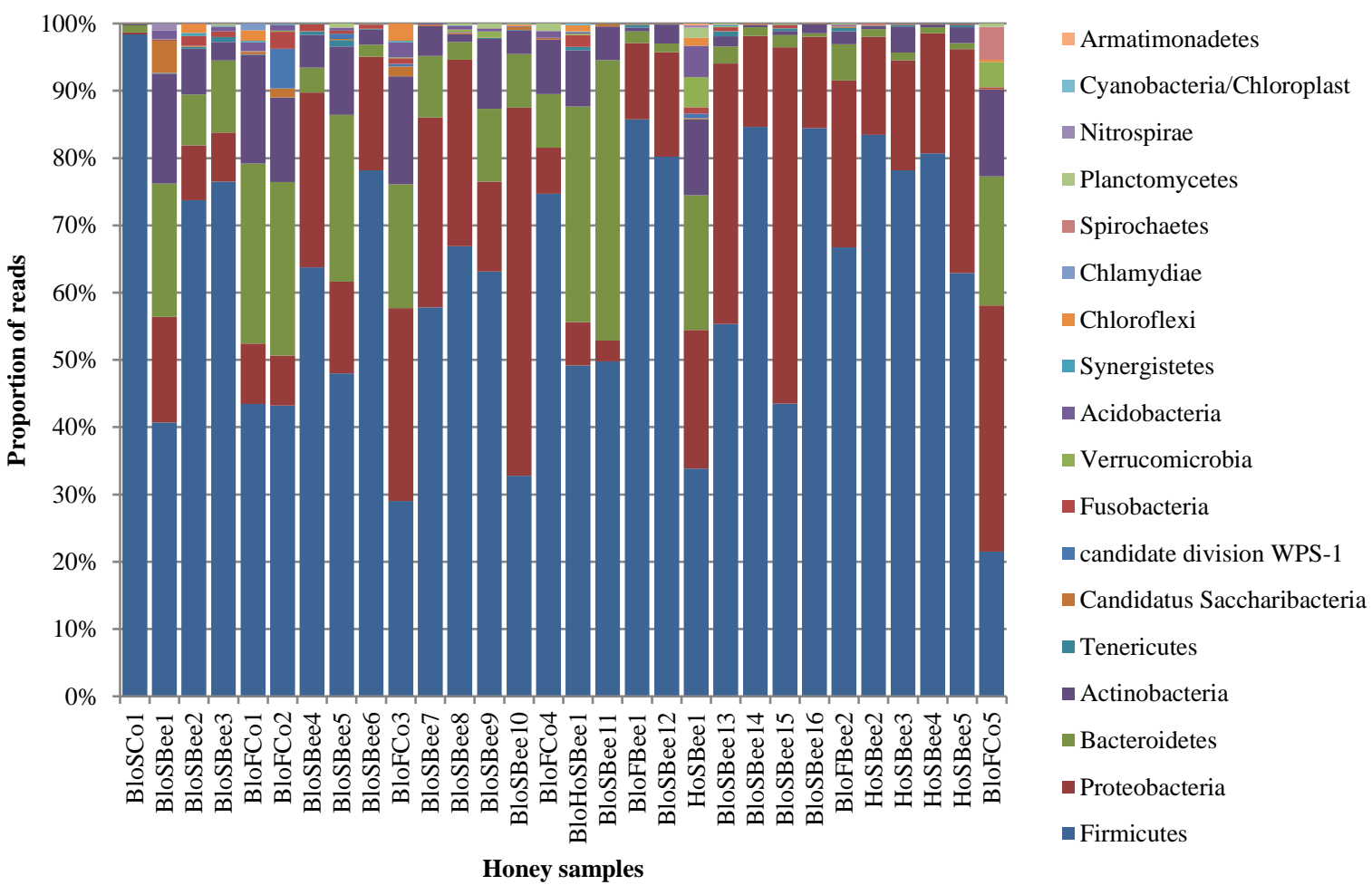

Figure 1 Proportion of reads assigned to bacterial phylum in honey samples

sample code (according to botanical, geographical origin and obtaining): first part: Blo - blossom, Ho - honeydew, BloHo - blended (blossomhoneydew); second part: S - Slovakian, F - foreign; third part: Bee - from beekeeper, Co - from commercial trade 


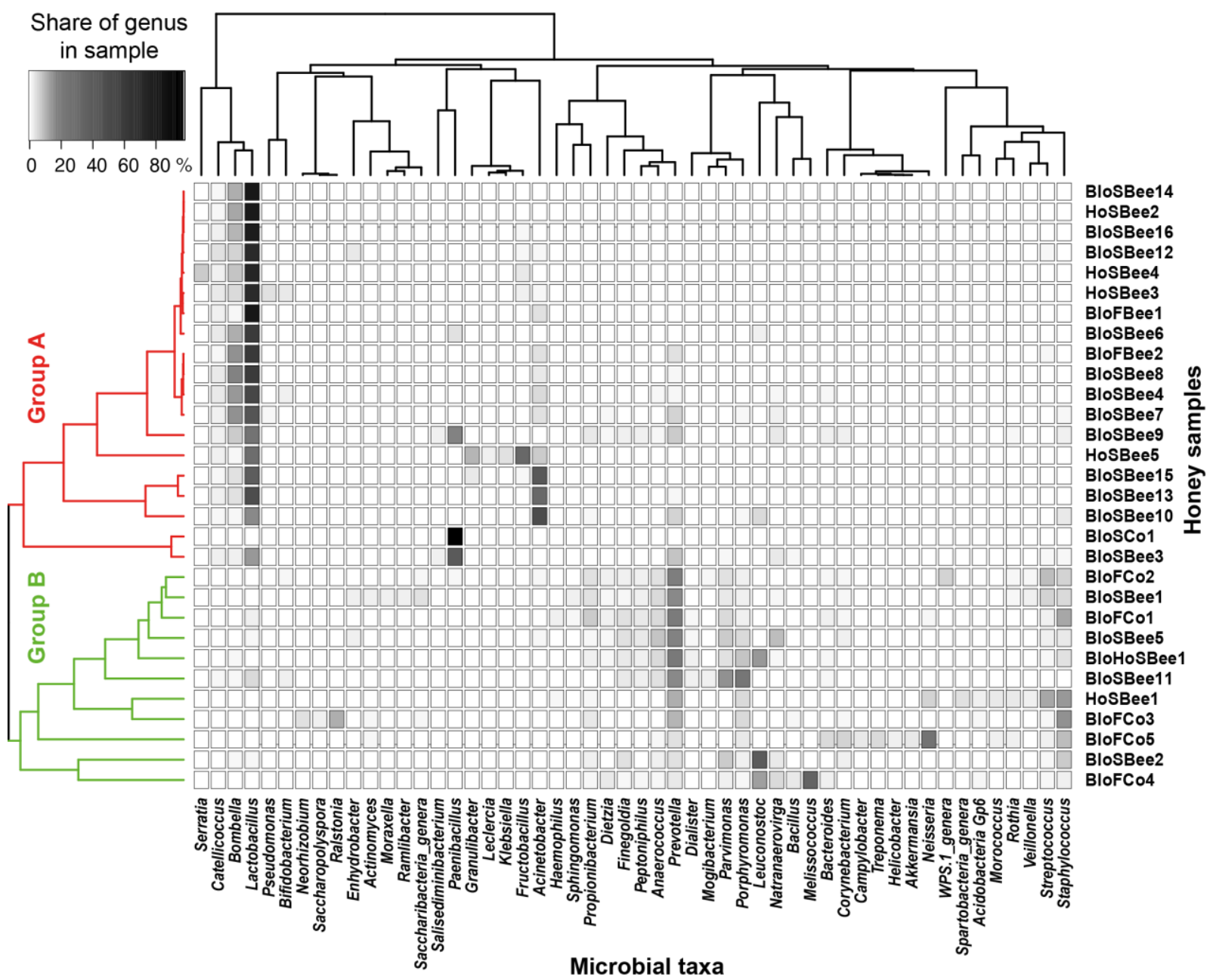

Figure 2 Bacterial genera with at least $2 \%$ share in any sample of honey and grouping of samples according bacteria occurrence sample code (according to botanical, geographical origin and obtaining): first part: Blo - blossom, Ho - honeydew, BloHo - blended (blossomhoneydew); second part: S - Slovakian, F - foreign; third part: Bee - from beekeeper, Co - from commercial trade

At presented heatmap (figure 2), honey samples were clustered to two big groups (clusters) - A and B - according to most common bacterial genera in samples.

The main factor of dividing into the group A and group B seems to be the freshnes of the sample, because the first 19 samples are blossom/honeydew honeys from beekeepers and commercial trade from the year 2018, except HoSBee3, which is from 2017 and BloSCo1, which is commercial. Next 11 samples originated from beekeepers from the year 2017 (except BloSBee 2, 11) and from commercial trade. Production year of commercial samples can be uncertain, because only packaging and/or expiration date are present at the label and in fact honey could be produced several years before packaging.

In group A consist of 19 honey samples, genera Lactobacillus was dominant followed by Bombella. In detailed analysis lactobacilli OTUs highest sequence similarity was found with Lactobacillus apinorum. Lactobacillus kunkeei is dominant LAB in honey stomach (crop) and fresh honey (Olofsson et Vásquez, 2008). L. apinorum, originated from the honey bee gut, is the second fructophilic LAB within the genus Lactobacillus (Maeno et al., 2017). Bombella apis was detected in midgut, crop and hive of honey bees and bumble bees (Bonilla-Rosso et Engel, 2018). Preliminary $\mathrm{LAB}$ were found in samples BloSBee 6, 9; BloHoSBee 1 and BloFCo 3, 5 by dilution plating method, but presence of LAB by lactobacilli detection was only in samples BloSBee 6 and 9. Although LAB do not survive in stored honey, it is likely that humans have been consuming viable $\mathrm{LAB}$ in fresh honey during and directly after honey hunts throughout human history (Olofsson et Vásquez, 2008). In 3 member subgroup (BloSBee 10, 13, 15)
Fructobacillus were very abundant while Paenibacillus were apparent in some samples. However, in sample BloSCo1, genus Lactobacillus was not detected, only genus Paenibacillus, which was dominant and in detailed analysis, it was identified as Paenibacillus alvei (from honey DNA followed by confirmation of culture DNA from the NA2 plate). Paenibacillus sp. were separated from the bacilli, they originated mainly from the soil and are common in the honey, but $P$. alvei is one of the secondary invaders of European Foulbrood (EFB) and P. larvae is the causative agent of American Foulbrood (AFB) (Generch, 2010).

Composition of microbial assemblage in group B (11 honey samples) samples was significantly different. Besides genera Leuconostoc and Melissococcus which were reported in honey, few other detected genera are not often in honeys or bees. Bacteria Melissococcus pluton causes the brood disease EFB, while some other organisms, like Paenibacillus alvei, P. apiaries, Brevibacillus laterosporus, Enterococcus faecalis or Bacterium eurydice are present as secondary invaders of dead brood (Shimanuki et Knox, 2000). Presence of genera like Prevotella, Staphylococcus or Porphyromonas suggests contamination of these samples, during the extracting, processing and/or storage of the samples. On the other side, last time, presence of Neisseria sp., which mostly known as human pathogen, were confirmed in gut of bees and bumblebees (Kwong et Moran, 2013). 


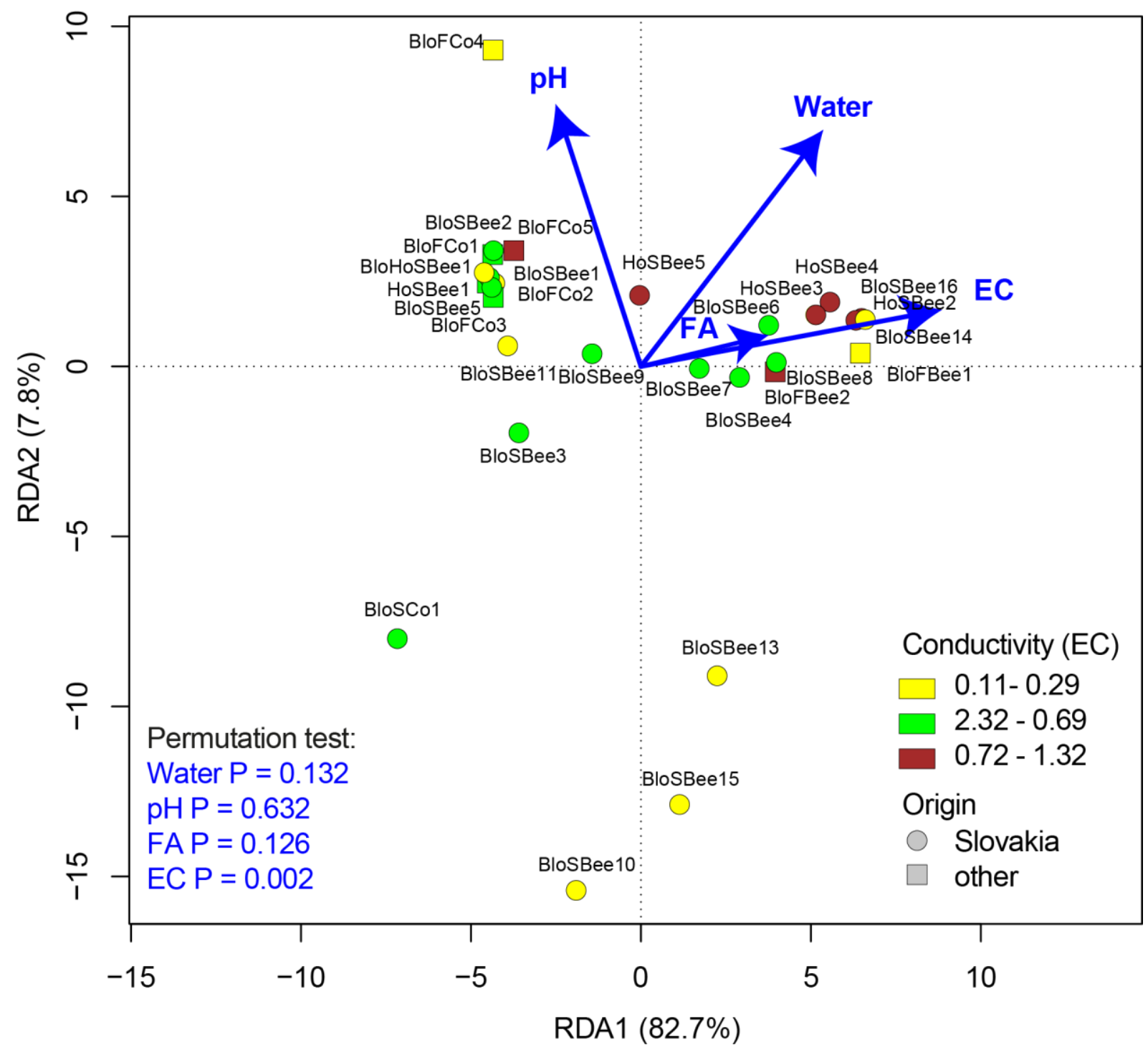

Figure 3 RDA scatterplot from analysis of physico-chemical properties and microbial assemblage of honey sample code (according to botanical, geographical origin and obtaining): first part: Blo - blossom, Ho - honeydew, BloHo - blended (blossom-honeydew); second part: S - Slovakian, F - foreign; third part: Bee - from beekeeper, Co - from commercial trade; FA - free acidity, EC - electrical conductivity

Microbial assemblage of honey is connected mainly to the microbiome of bees. Honey seems to be a suitable indicator of surrounding during the honey production, processing and storage. Relation of physic-chemical attributes and microbia assemblage using redundancy analysis showed electrical conductivity as main factor related to variation in honey microbiome (figure 3). First RDA factor explained majority of variance $(82.7 \%)$ in microbial assemblage. This factor was in strong correlation $(\mathrm{R} 2=0.69)$ with EC. Permutation test approved EC as the only one significant physico-chemical attribute $(\mathrm{P}=0.003)$ among tested. According to Bogdanov et Martin (2002), EC is suitable parameter for evaluation differentiation between blossom and honeydew honeys as well as for unifloral honeys. As EC is strongly depended on source of honey, probably the source is important determinant of microbial assemblage at least the same that bees themselves.

\section{CONCLUSION}

Overall, electrical conductivity was the main parameter, which was connected with the botanical origin. Viable counts of microorganisms could be influenced by the water content as well as freshness of the sample. We found sporulating aerobic microorganisms and yeasts as the most often occurring viable microbes. However, metagenomic analysis gave us interesting view into the presence of bacterial DNA in the honey. Bacterial diversity in the honey samples indicated main differences between the Slovak and Swiss fresh samples (produced in 2018) and the older ones (produced in 2017) together with commercial samples from Slovakia and foreign countries. In general, lactobacilli were dominant in Slovakian and Swiss fresh honeys. In older samples, Prevotella sp. and other representatives probably originating in human contamination during the honey extracting and processing were dominant. Surprisingly, the spectrum of identified bacterial genera was broad.
Probably, most of them are not in viable state as honey represents a suitable environment for keeping of DNA and RNA intact. Subsequently, data from metagenomic analysis can provide us various information about surrounding during honey production, processing and storage.

Acknowledgments: The work was supported by the grant KEGA 025SPU-4/2019 and VEGA 1/0661/19. Authors would thank to prof. Radoslav Omelka from University of Constantine the Philosopher in Nitra for access to Next Generation Sequencer.

\section{REFERENCES}

AL-FARSI, M., AL-BELUSHI, S., AL-AMRI, A., AL-HADHRAMI, A., ALRUSHEIDI, M., AL-ALAWI, A. 2018. Quality evaluation of Omani honey. Food Chemistry, 262(2018), 162-167. http://doi.org/10.1016/j.foodchem.2018.04.104 AMIR, Y., YESLI, A., BENGANA, M., SADOUDI, R., AMROUCHE, T. 2010 Physico-chemical and microbiological assessment of honey from Algeria Electronic Journal of Environmental, Agricultural and Food Chemistry, 9(9), 1485-1494.

BOGDANOV, S., MARTIN, P. 2002. Honey Authenticity. Mitteilungen aus Lebensmitteluntersuchung und Hygiene, 93(3), 232-254.

BOGDANOV, S., RUOFF, K., PERSANO ODDO, L. 2004. Physico-chemica methods for the characterisation of unifloral honeys: a review. Apidologie, 35(1), S4-S17. http://doi.org/10.1051/apido:2004047

BONILLA-ROSSO, G., ENGEL, P. 2018. Functional roles and metabolic niches in the honey bee gut microbiota. Current Opinion in Microbiology, 43(2018), 6976. http://doi.org/10.1016/j.mib.2017.12.009 
BOVO S., RIBANI A., UTZERI V. J., SCHIAVO G., BERTOLINI F., FONTANESI L. 2018. Shotgun metagenomics of honey DNA: Evaluation of a methodological approach to describe a multi-kingdom honey bee derived environmental DNA signature. PLOS ONE, 13(10), e0205575 http://doi.org/10.1371/journal.pone.0205575

BUŠOVÁ, M., KOUŘIMSKÁ, L. 2018. Comparing the quality of honey from beekeepers and honey from the market chain. Potravinarstvo Slovak Journal of Food Sciences, 12(1), 364-371. http://doi.org/10.5219/909

CAPORASO, J. G., LAUBER, C. L., WALTERS, W. A., BERG-LYONS, D. LOZUPONE, C. A., TURNBAUGH, P. J., FIERER, N., KNIGHT, R.. 2011 Global patterns of $16 \mathrm{~S}$ rRNA diversity at a depth of millions of sequences per sample. Proceedings of the national academy of sciences, 108(suppl. 1), 45164522. https://doi.org/10.1073/pnas. 1000080107

COUNCIL DIRECTIVE 2001/110/EC of 20 December 2001 relating to honey.

COOPER, R. 2014. Honey as an effective antimicrobial treatment for chronic wounds: is there a place for it in modern medicine? Journal of Chronic Wound Care Management and Research, 2014(1), 15-22. http://doi.org/10.2147/CWCMR.S46520

DROZD P. 2010. ComEcoPaC-Community Ecology Parameter Calculator. Version 1.

ESCUREDO, O., FERNÁNDEZ-GONZÁlEZ, M., SEIJO, M. C. 2012 Differentiation of Blossom Honey and Honeydew Honey from Northwest Spain. Agriculture, 2(4), 25-37. http://doi.org/10.3390/agriculture2010025

GENERSCH, E. 2010. American Foulbrood in honeybees and its causative agent Paenibacillus larvae. Journal of Invertebrate Pathology, 103 (suppl.), S10-S19. http://doi.org/10.1016/j.jip.2009.06.015

GILLIAM, M., MOFFETT, J. O., KAUFFELD, N. M. 1983. Examination of flora nectar of citrus, cotton, and Arizona Desert plants for microbes. Apidologie, 14(4), 299-302. http://doi.org/10.1051/apido:19830403

GUZIY, S., ŠEDIK, P., HORSKA, E. 2017. Comparative study of honey consumption in Slovakia and Russia. Potravinarstvo Slovak Journal of Food Sciences, 11(1), 472-479. http://doi.org/10.5219/784

GULFRAZ, M., IFTIKHAR, F., IMRAN, M. ZEENAT, A, ASIF, S, SHAH, I. 2011. Compositional analysis and antimicrobial activity of various honey types of Pakistan. International Journal of Food Science \& Technology, 46(2), 263-267. http://doi.org/10.1111/j.1365-2621.2010.02488.x

HANDELSMAN, J. 2004. Metagenomics: Application of Genomics to Uncultured Microorganisms. Microbiology and Molecular Biology Reviews, 68(4), 669-685. http://doi.org/10.1128/MMBR.68.4.669-685.2004

IHC. 2009. Harmonised methods of the International Honey Commission. 63 p.

ISRAILI, Z. H. 2014. Antimicrobial Properties of Honey. American Journal of Therapeutics, 21(4), 304-323. http://doi.org/10.1097/MJT.0b013e318293b09b

KWONG, W. K., MORAN, N. A. 2013. Cultivation and characterization of the gut symbionts of honey bees and bumble bees: description of Snodgrassella alvi gen. nov., sp. nov., a member of the family Neisseriaceae of the Betaproteobacteria, and Gilliamella apicola gen. nov., sp. nov., a member of Orbaceae fam. nov., Orbales ord. nov., a sister taxon to the order 'Enterobacteriales' of the Gammaproteobacteria. International Jornal of Systematic and Evolutionary Microbiology, 63(6), 2008-2018 http://doi.org/10.1099/ijs.0.044875-0

MAENO, S., DICKS, L., NAKAGAWA, J., ENDO, A. 2017. Lactobacillu apinorum belongs to the fructophilic lactic acid bacteria. Bioscience of Microbiota Food and Health, 36(4), 147-149. http://doi.org/10.12938/bmfh.17-008

MAES, P. W., RODRIGUES, P. A. P., OLIVER, R., MOTT, B. M., ANDERSON K. E. 2016. Diet-related gut bacterial dysbiosis correlates with impaired development, increased mortality and Nosema disease in the honeybee (Apis mellifera). Molecular Ecology, 25(21), 5439-5450. http://doi.org/10.1111/mec.13862

MARTINS, H. M., MARTINS, M. L., BERNARDO, F. M. A. 2003. Bacillaceae spores, fungi and aflatoxins determination in honey. Revista Portuguesa de Ciências veterinárias, 98(546), 85-88

MEDO, J., ŽIAROVSKÁ, J., MEDOVÁ, J., JAVOREKOVÁ, S., KYSEL, M., HRICOVÁ, A. 2018. Endophytic bacterial diversity decrease in amaranth mutant lines after radiation mutagenesis. Cereal Chemistry, 95(1), 109-116. http://doi.org/10.1002/cche.10006

OKSANEN, J., BLANCHET, F. G., KINDT, R., LEGENDRE, P., MINCHIN, P. R., O'HARA, R., SIMPSON, G. L., SOLYMOS, P., STEVENS, M. H. H. WAGNER, H. 2013. Package 'vegan'. Community ecology package. Version 2. OLAITAN, P. B., ADELEKE, O. E., IYABO, O. O. 2007. Honey: a reservoir for microorganisms and an inhibitory agent for microbes. African Health Sciences, 7(3), 159-165

OLOFSSON, T. C., VÁSQUEZ, A. 2008. Detection and Identification of a Novel Lactic Acid Bacterial Flora Within the Honey Stomach of the Honeybee Apis mellifera. Current Microbiology, 57(4), 356-363. http://doi.org/10.1007/s00284$\underline{008-9202-0}$

ROGNES, T., FLOURI, T., NICHOLS, B., QUINCE, C., MAHÉ, F. 2016 VSEARCH: a versatile open source tool for metagenomics. PeerJ, 4(2016), e2584 http://doi.org/10.7717\%2Fpeerj.2584
SHIMANUKI, H., KNOX, D. A. 2000. Diagnosis of Honey Bee Diseases: Agriculture Handbook no. 690. Washington : U. S. Department of Agriculture, 61

SNOWDON, J. A., CLIVER, D. O. 1996. Microorganisms in honey. International Journal of Food Microbiology, 31(1-3), 1-26. http://doi.org/10.1016/0168 1605(96)00970-1

SEDIK, P., POCOL, C. B., HORSKA, E., FIORE, M. 2019. Honey: food or medicine? A comparative study between Slovakia and Romania. British Food Journal, 121(6), 1281-1297. http://doi.org/10.1108/BFJ-12-2018-0813

TEAM R. 2013. R: A language and environment for statistical computing.

TOLBA, O., EARLE, J. A. P., MILLAR, B. CH., ROONEY, P. J., MOORE, J. E 2007. Speciation of Bacillus spp. in honey produced in Northern Ireland by employment of $16 \mathrm{~S}$ rDNA PCR and automated DNA sequencing techniques. World Journal of Microbiology and Biotechnology, 23(12), 1805-1808. http://doi.org/10.1007/s11274-007-9425-4

VĚTROVSKÝ, T., BALDRIAN, P., MORAIS, D. 2018. SEED 2: a user-friendly platform for amplicon high-throughput sequencing data analyses. Bioinformatics, 34(13), 2292-2294. http://doi.org/10.1093/bioinformatics/bty071

VIUDA-MARTOS, M., RUIZ-NAVAJAS, Y., FERNÁNDEZ-LÓPEZ, J. PÉREZ-ÁLVAREZ, J. A. 2008. Functional Properties of Honey, Propolis, and Royal Jelly. Journal of Food Science, 73(9), R117-R124 http://doi.org/10.1111/j.1750-3841.2008.00966.x

WANG, Q., GARRITY, G. M., TIEDJE, J. M., COLE, J. R. 2007. Naïve Bayesian classifier for rapid assignment of rRNA sequences into the new bacteria taxonomy. Applied and Environmental Microbiology, 73(16), 5261-5267. http://doi.org/10.1128/AEM.00062-07

ZHAO, S., GUO, Y., SHENG, Q., SHYR, Y. 2014. Advanced Heat Map and

Clustering Analysis Using Heatmap3. BioMed Research International, 2014, ID 986048, 1-6. http://doi.org/10.1155/2014/986048

ZIELIŃSKA， S., WESEŁOWSKA, M., BILEK, M., KANIUCZAK, J. DŻUGAN, M. 2014. The saccharide profile of polish honeys depending on their botanical origin. Journal of Microbiology, Biotechnology and Food Sciences, 3(5), 387-390. 